\title{
CUMULANTS AS ITERATED INTEGRALS
}

\author{
FRANZ LEHNER
}

\begin{abstract}
A formula expressing cumulants in terms of iterated integrals of the distribution function is derived. It generalizes results of Jones and Balakrishnan who computed expressions for cumulants up to order 4 .
\end{abstract}

\section{INTRODUCTION}

The expectation of a random variable can be computed in many ways. One method involving only the distribution function is obtained by careful partial integration and looks as follows:

$$
\mathbf{E} X=\int_{0}^{\infty}(1-F(t)) d t-\int_{-\infty}^{0} F(t) d t ;
$$

a similar formula holds for the variance, which can be written as the following double integral:

$$
\operatorname{Var}(X)=2 \iint_{-\infty<t_{1}<t_{2}<\infty} F\left(t_{1}\right)\left(1-F\left(t_{2}\right)\right) d t_{1} d t_{2} .
$$

Analogues of these formulas expressing the third an fourth cumulants (skewness and kurtosis) in terms of iterated integrals of the distribution function were computed some time ago by Jones and Balakrishnan [7]. The proof relied on ad hoc partial integration, see also [2], where similar formulas for mean differences are considered.

The aim of the present note is a generalization of these expressions to cumulants of arbitrary order, resulting in a formula resembling the well-known Möbius inversion formula, which expresses cumulants in terms of moments.

The paper is organized as follows. After a short introduction to cumulants in Section 2 and a review of partitions and shuffles in Section 3 we give two proofs of the main result. The first one using an elementary identity for the Volterra integral operator and Chen's shuffle formula for multiple integrals is contained in Section 4 . In the concluding Section 5 we give another proof based on a formula for multivariate cumulants due to Block and Fang.

\section{Moments And Cumulants}

Let $X$ be a random variable with distribution function $F(x)=P(X \leq x)=\int_{-\infty}^{x} d F(t)$. Its moments are the numbers

$$
m_{n}=\mathbf{E} X^{n}=\int_{-\infty}^{\infty} t^{n} d F(t)
$$

Date: May 20, 2009.

1991 Mathematics Subject Classification. 60E05;62E10, 62E15.

Key words and phrases. moments, cumulants, shuffle, Hoeffding's lemma, iterated integral. 
Under some assumptions the sequence of moments contains the complete information about the distribution of $X$. It can be collected in the exponential moment generating function (formal Fourier-Laplace transform)

$$
\mathcal{F}_{X}(z)=\mathbf{E} e^{z X}=\sum_{n=0}^{\infty} \frac{m_{n}}{n !} z^{n}=1+\frac{m_{1}}{1 !} z+\frac{m_{2}}{2 !} z^{2}+\cdots
$$

and the Taylor coefficients of the formal logarithm of the m.g.f.

$$
\log \mathcal{F}_{X}(z)=\sum_{n=1}^{\infty} \frac{\kappa_{n}}{n !} z^{n}
$$

are called the cumulants. The first two are expectation and variance

$$
\begin{aligned}
& \kappa_{1}=m_{1}=\mathbf{E} X \\
& \kappa_{2}=m_{2}-m_{1}^{2}=\operatorname{Var} X
\end{aligned}
$$

and after rescaling the following two are the skewness $\kappa_{3} / \kappa_{2}^{3 / 2}$ and the kurtosis $\kappa_{4} / \kappa_{2}^{2}$.

The cumulants carry the same information as the moments but for many purposes in a better digestible form, e.g., after a translation the moments behave like

$$
m_{n}(X+\tau)=\sum_{k=0}^{n}\left(\begin{array}{l}
n \\
k
\end{array}\right) \tau^{n-k} m_{k}(X)
$$

while the cumulants are

$$
\kappa_{n}(X+\tau)= \begin{cases}\tau+\kappa_{1}(X) & n=1 \\ \kappa_{n}(X) & n \geq 2\end{cases}
$$

For this reason the cumulants are sometimes called the semi-invariants of $X$. The most important property of the cumulants is the identity

$$
\kappa_{n}(X+Y)=\kappa_{n}(X)+\kappa_{n}(Y)
$$

if $X$ and $Y$ are independent.

\section{Partitions And Shuffles}

There is also a combinatorial formula expressing the cumulants as polynomials of the moments. A set partition of order $n$ is set

$$
\pi=\left\{B_{1}, B_{2}, \ldots, B_{p}\right\}
$$

of of disjoint subsets $B_{i} \subseteq\{1,2, \ldots, n\}$, called blocks, whose union is $\{1,2, \ldots, n\}$. Denote $\Pi_{n}$ the set of all $n$-set partitions. It is a lattice under the refinement order

$$
\pi \leq \sigma \Longleftrightarrow \text { every block of } \pi \text { is contained in a block of } \sigma,
$$

with minimal element $\hat{0}_{n}=\{\{1\},\{2\}, \ldots,\{n\}\}$ and maximal element $\hat{1}_{n}=\{\{1,2, \ldots, n\}\}$. Each set partition $\pi \in \Pi_{n}$ determines a number partition, called its type, $\lambda(\pi)=1^{k_{1}} 2^{k_{2}} \cdots n^{k_{n}} \vdash$ $n$ where $k_{j}$ is the number of blocks $B \in \pi$ of size $|B|=j$. For the combinatorial identities below we will employ the following conventions. For a partition $\lambda=1^{k_{1}} 2^{k_{2}} \cdots n^{k_{n}} \vdash n$ we abbreviate $\lambda !=1 !^{k_{1}} 2 !^{k_{2}} \cdots n !^{k_{n}}$ and for a sequence $\left(a_{n}\right)_{n \in \mathbf{N}}$ of numbers we denote

$$
a_{\lambda}=\prod a_{j}^{k_{j}}
$$


similarly for a set partition $\pi$ we let

$$
a_{\pi}=a_{\lambda(\pi)}=\prod_{B \in \pi} a_{|B|} .
$$

Given a partition $\lambda \vdash n$, the number of set partitions $\pi \in \Pi_{n}$ with $\lambda(\pi)=\lambda$ is equal to the Faa di Bruno coefficient

$$
\left\{\begin{array}{l}
n \\
\lambda
\end{array}\right\}=\#\{\pi: \pi \sim \lambda\}=\frac{n !}{1 !^{k_{1}} 2 !^{k_{2}} \cdots n !{ }^{k_{n}} k_{1} ! k_{2} ! \cdots k_{n} !}
$$

The well known moment-cumulant formula says

$$
\kappa_{n}=\sum_{\pi \in \Pi_{n}} m_{\pi} \mu\left(\pi, \hat{1}_{n}\right)
$$

where $\mu$ is the Möbius function on $\Pi_{n}$. Its values only depend on $\lambda(\pi)=1^{k_{1}} 2^{k_{2}} \cdots n^{k_{n}}$, namely

$$
\mu\left(\pi, \hat{1}_{n}\right)=\mu_{\lambda(\pi)}=\prod_{j=1}^{n}\left((-1)^{j}(j-1) !\right)^{k_{j}}
$$

Using the Faa Di Bruno coefficients (3.1) the moment-cumulant formula can be condensed to

$$
\kappa_{n}=\sum_{\lambda \vdash n}\left\{\begin{array}{l}
n \\
\lambda
\end{array}\right\} m_{\lambda} \mu_{\lambda}
$$

Definition 3.1. Let $a=\left(a_{1}, a_{2}, \ldots, a_{m}\right)$ and $b=\left(b_{1}, b_{2}, \ldots, b_{m}\right)$ be two finite sequences. A shuffle of $a$ and $b$ is a pair of order preserving injective maps $\varphi: a \rightarrow\{1, \ldots, m+n\}$ and $\psi: b \rightarrow\{1, \ldots, m+n\}$ with disjoint images. When the two sequences are thought of as two decks of cards, this corresponds to putting the two decks together in such a way that the relative order in the individual decks is preserved, the card $a_{i}$ (resp. $b_{j}$ ) being put in position $\varphi\left(a_{i}\right)$ (resp. $\psi\left(b_{j}\right)$ ). The result of the shuffle is the sequence, where each $i$ is replaced by the symbol $\varphi^{-1}(i)$ (or $\left.\psi^{-1}(i)\right)$. Denote $\amalg(m, n)$ the set of shuffles of the sequences $(1,2, \ldots, m)$ and $(m+1, m+2, \ldots, m+n)$. Shuffles of multiple sequences are defined accordingly and for a partition $\lambda \vdash n$ denote $\amalg(\lambda)$ the set of shuffles of disjoint sequences with cardinalities given by $\lambda$.

Each shuffle is uniquely determined by the subsets the individual sequences are mapped to and thus the number of shuffles is equal to the number of ways of picking these subsets, i.e., the multinomial coefficient

$$
\# \amalg(\lambda)=\left(\begin{array}{l}
n \\
\lambda
\end{array}\right)
$$

On the other hand each shuffle $\tau \in \amalg(\lambda)$ determines a partition $\pi$ of $\{1,2, \ldots, n\}$ of type $\lambda$. However different shuffles may determine the same partition $\pi$, if $\lambda$ contains entries of the same size. Therefore we have the identity

$$
\# \amalg(\lambda)=k_{1} ! k_{2} ! \cdots k_{n} !\left\{\begin{array}{l}
n \\
\lambda
\end{array}\right\}
$$

which will lead to an interesting cancellation later. 


\section{ITERATEd InTEGRALS}

Definition 4.1. The Volterra operator is the integral operator

$$
V f(x)=\int_{-\infty}^{x} f(t) d t
$$

defined for suitable integrable functions $f: \mathbf{R} \rightarrow \mathbf{R}$; its powers are defined recursively by

$$
V^{n} f(x)=\int_{-\infty}^{x}\left(V^{n-1} f\right)(t) d t
$$

It was first observed by Chen [4] and Ree [9] that the recursively defined iterated integrals

$$
\alpha_{i_{1}, i_{2}, \ldots, i_{n}}(t)=\int_{a}^{t} \alpha_{i_{1}, i_{2}, \ldots, i_{n-1}}(u) d \alpha_{i_{n}}(u)
$$

which can be written as

$$
\iint_{a<t_{1}<t_{2}<\cdots t_{n}} \cdots \int_{i_{1}}\left(t_{1}\right) d \alpha_{i_{2}}\left(t_{2}\right) \cdots d \alpha_{i_{n}}\left(t_{n}\right)
$$

satisfy the shuffle relations

$$
\alpha_{i_{1}, i_{2}, \ldots, i_{m}}(t) \alpha_{j_{1}, j_{2}, \ldots, j_{n}}(t)=\sum_{\sigma \in \amalg\left(\left\{i_{1}, \ldots, i_{m}\right\},\left\{j_{1}, \ldots, j_{n}\right\}\right)} \alpha_{\sigma}(t)
$$

For the Volterra operator this means that for example

$$
V^{m} f(x) V^{n} f(x)=\sum_{\tau \in \amalg(m, n)} \iint_{t_{1}<\cdots<t_{m+n}} \ldots \int f_{\tau}\left(t_{1}, \ldots, t_{m+n}\right) d t_{1} d t_{2} \cdots d t_{m+n} ;
$$

where $f_{\tau}\left(t_{1}, \ldots, t_{m+n}\right)=f\left(t_{\varphi(1)}\right) f\left(t_{\psi(1)}\right)$ with $\varphi$ and $\psi$ as in Definition 3.1.

Note that if $f(t)$ is a probability density function, then the corresponding distribution function is given by

$$
F(x)=V f(x)
$$

We denote $F^{[n]}(x)=V F^{[n-1]}(x)=V^{n+1} f(x)$ where $F^{[0]}(x)=F(x)=\int_{-\infty}^{x} d F(t)$. This notation slightly differs from [2]. Then one can easily show by induction that these integrals are truncated moments.

Lemma 4.2 .

$$
F^{[n]}(\tau)=\frac{1}{n !} \int_{-\infty}^{\tau}(\tau-t)^{n} d F(t)=\frac{1}{n !} \mathbf{E}(\tau-X)_{+}^{n}
$$

Before proceeding further, assume for the moment that the support of $d F$ is bounded and that $\tau$ is an upper bound. Then $Y=X-\tau$ has moments

$$
y_{n}=\mathbf{E}(X-\tau)^{n}=(-1)^{n} n ! F^{[n]}(\tau)=\sum_{k=0}^{n}\left(\begin{array}{l}
n \\
k
\end{array}\right)(-\tau)^{n-k} m_{k}
$$

and because of (2.1) the cumulants are

$$
\kappa_{n}(X)= \begin{cases}\kappa_{1}(Y)+\tau & n=1 \\ \kappa_{n}(Y) & n \geq 2\end{cases}
$$


Thus we can express the cumulants of order $n \geq 2$ by a moment-cumulant type formula in terms of $y_{n}=(-1)^{n} n ! V^{n} F$ :

$$
\kappa_{n}(X)=\sum_{\pi \in \Pi_{n}} y_{\pi} \mu\left(\pi, \hat{1}_{n}\right)
$$

After some shuffling (in the literal sense!) this simplifies to the following formula.

\section{Theorem 4.3.}

$$
\kappa_{n}=(-1)^{n} n ! \iint_{t_{1}<t_{2}<\cdots<t_{n}} \ldots \int_{\pi \in \Pi_{n}} F_{\pi}\left(t_{1}, t_{2}, \ldots, t_{n}\right) \mu\left(\pi, \hat{1}_{n}\right) d t_{1} d t_{2} \cdots d t_{n}
$$

where $F_{\pi}\left(t_{1}, t_{2}, \ldots, t_{n}\right)=\prod_{B \in \pi} F\left(t_{\alpha(B)}\right)$ where $\alpha(B)$ denotes the first (i.e., smallest) element of a block $B$.

Note that the higher order cumulants do not depend on $\tau$ and thus the formula also holds if the support of $X$ is unbounded.

Remark 4.4. It may be hoped that this formula provides some insight to Rota's problem of the cumulants [10], namely to find a collection of inequalities which are necessary and sufficient for a number sequence to be the cumulant sequence of some probability distribution. One advantage of formula (4.2) is the fact that all terms appearing in the sum are either nonnegative or nonpositive, regardless which particular probability distribution is considered, in contrast to the moments in Schützenberger's formula (3.2).

Proof. Assume first that the support of $X$ is bounded. Starting from (4.1), or rather (3.3), we have

$$
\begin{aligned}
\kappa_{n} & =\sum_{\lambda \vdash n}\left\{\begin{array}{l}
n \\
\lambda
\end{array}\right\} y_{\lambda} \mu_{\lambda} \\
& =(-1)^{n} \sum_{\lambda \vdash n}\left\{\begin{array}{l}
n \\
\lambda
\end{array}\right\} \lambda ! F^{[\lambda]}(\tau) \mu_{\lambda}
\end{aligned}
$$

and by Chen's lemma this is equal to

$$
\left.=(-1)^{n} \sum_{\lambda \vdash n}\left\{\begin{array}{l}
n \\
\lambda
\end{array}\right\} \lambda ! \sum_{\sigma \in \amalg(\lambda)} \iint_{-\infty<t_{1}<t_{2}<\cdots<t_{n}<\tau} \ldots \int_{\sigma} F_{1}, \ldots, t_{n}\right) d t_{1} d t_{2} \cdots d t_{n} \mu_{\lambda} .
$$

Since $F_{\sigma}$ only depends on the partition determined by $\sigma$, we can collect equal terms to get

$$
\begin{aligned}
& =(-1)^{n} \sum_{\lambda \vdash n}\left\{\begin{array}{l}
n \\
\lambda
\end{array}\right\} \lambda ! k_{1} ! k_{2} ! \ldots k_{n} ! \sum_{\pi \sim \lambda} \iint_{-\infty<t_{1}<t_{2}<\cdots<t_{n}<\tau} \ldots \int_{\pi} F_{\pi}\left(t_{1}, \ldots, t_{n}\right) d t_{1} d t_{2} \cdots d t_{n} \mu_{\lambda} \\
& =(-1)^{n} n ! \sum_{\pi \in \Pi_{n}} \iint_{-\infty<t_{1}<t_{2}<\cdots<t_{n}<\tau} \ldots \int_{\pi} F_{1}\left(t_{1}, \ldots, t_{n}\right) d t_{1} d t_{2} \cdots d t_{n} \mu\left(\pi, \hat{1}_{n}\right) .
\end{aligned}
$$

Since the final formula does not depend on the chosen integration bound $\tau$, we may let it go to infinity and the formula holds for arbitrary distribution functions. 
Remark 4.5. Using the recursive structure of the partition lattice, we can partially factorize the integrand of (4.2). Each partition $\pi \in \Pi_{n}$ can be constructed from a unique partition $\pi^{\prime} \in \Pi_{n-1}$ by either adjoining $\{n\}$ as a separate block or by joining $n$ to one of the blocks of $\pi^{\prime}$. Assume that $\pi^{\prime}$ has $k$ blocks. In the first case the number of blocks is increased to $k+1$ and the Möbius function, which depends on the number of blocks, becomes $\mu\left(\pi, \hat{1}_{n}\right)=-k \mu\left(\pi^{\prime}, \hat{1}_{n-1}\right)$. The integrand changes simply to $F_{\pi}(t)=F_{\pi^{\prime}}(t) F\left(t_{n}\right)$.

In the second case the number of blocks remains the same and also the Möbius function and integrand stays the same. However there are $k$ possible ways to join $n$ to a block of $\pi^{\prime}$. Thus we can write

$$
\begin{aligned}
\kappa_{n}(X)=(-1)^{n} n ! \iint_{t_{1}<t_{2}<\cdots<t_{n}} \ldots \int_{k=1}^{n-1} \sum_{\pi^{\prime} \in \Pi_{n-1, k}}\left(-k F_{\pi^{\prime}}\left(t_{1}, t_{2}, \ldots, t_{n-1}\right) F\left(t_{n}\right)\right. \\
\quad+k F_{\pi^{\prime}}\left(t_{1}, t_{2}, \ldots, t_{n-1}\right) \mu\left(\pi^{\prime}, \hat{1}_{n-1}\right) d t_{1} d t_{2} \cdots d t_{n} \\
=(-1)^{n} n ! \iint_{t_{1}<t_{2}<\cdots<t_{n}} \ldots \sum_{\pi \in \Pi_{n-1}}|\pi| F_{\pi}\left(t_{1}, t_{2}, \ldots, t_{n-1}\right)\left(1-F\left(t_{n}\right)\right) \mu\left(\pi, \hat{1}_{n-1}\right) d t_{1} d t_{2} \cdots d t_{n}
\end{aligned}
$$

The number of integration variables can still be reduced by two as discussed in [6]. For this purpose we introduce the so-called mean redidual life functions of Barlow and Proschan [1], namely

$$
\begin{aligned}
& R(y)=\mathbf{E}(X \mid X>y)-y=\frac{\int_{y}^{\infty}(1-F(t)) d t}{1-F(y)} \\
& P(y)=y-\mathbf{E}(X \mid X<y)=\frac{\int_{-\infty}^{y} F(t) d t}{F(y)}
\end{aligned}
$$

and obtain

$$
\kappa_{n}(X)=(-1)^{n} n ! \iint_{t_{2}<\cdots<t_{n-1}} \ldots \int_{\pi \in \Pi_{n-1}}|\pi| P\left(t_{2}\right) F_{\pi}\left(t_{2}, t_{2}, \ldots, t_{n-1}\right)\left(1-F\left(t_{n-1}\right)\right) R\left(t_{n-1}\right) \mu\left(\pi, \hat{1}_{n-1}\right) d t_{2} d t_{3} \cdots d t_{n}
$$

because $F_{\pi}\left(t_{1}, t_{2}, \ldots, t_{n-1}\right)$ always contains $F\left(t_{1}\right)$ as a factor.

\section{HöFFDing's FORMUla AND MULTIVARIATE CUMUlAnts}

In this section we give another proof of Theorem 4.3 using an extension of Höffding's formula due to Block and Fang [3]. Multivariate cumulants are defined as coefficients of multivariate Fourier transforms, namely

$$
\kappa_{n}\left(X_{1}, X_{2}, \ldots, X_{n}\right)==\left.\frac{\partial}{\partial z_{1}} \cdots \frac{\partial}{\partial z_{n}} \log \mathbf{E} e^{z_{1} X_{1}+\cdots+z_{n} X_{n}}\right|_{z_{1}=\cdots=z_{n}=0}
$$

The univariate cumulants in Section 2 correspond to the case $X_{1}=X_{2}=\cdots=X_{n}=X$. As an example, the second cumulant is the covariance

$$
\kappa_{2}\left(X_{1}, X_{2}\right)=\operatorname{Cov}\left(X_{1}, X_{2}\right)=\mathbf{E} X_{1} X_{2}-\mathbf{E} X_{1} \mathbf{E} X_{2} .
$$

In general the multivariate analogue of $(3.2)$ is

$$
\kappa_{n}\left(X_{1}, X_{2}, \ldots, X_{n}\right)=\sum_{\pi \in \Pi_{n}} m_{\pi}\left(X_{1}, X_{2}, \ldots, X_{n}\right) \mu\left(\pi, \hat{1}_{n}\right)
$$


where for a partition $\pi \in \Pi_{n}$ we denote

$$
m_{\pi}\left(X_{1}, X_{2}, \ldots, X_{n}\right)=\prod_{B \in \pi} \mathbf{E} \prod_{i \in B} X_{i}
$$

Höffding's formula [5, 8] gives an alternative expression for the covariance in terms of distribution functions:

$$
\kappa_{2}\left(X_{1}, X_{2}\right)=\iint\left(F\left(t_{1}, t_{2}\right)-F_{1}\left(t_{1}\right) F_{2}\left(t_{2}\right)\right) d t_{1} d t_{2}
$$

where $F\left(t_{1}, t_{2}\right)=\mathbf{P}\left(X_{1} \leq t_{1} \wedge X_{2} \leq t_{2}\right)$ is the joint distribution function and $F_{1}$ and $F_{2}$ are the marginal distribution functions of $X_{1}$ and $X_{2}$. From this it is easy to derive (1.1).

We shall use an extension of Höffding's formula to cumulants of all orders, due to Block and Fang [3] from which Theorem 4.3 follows as a corollary. For the reader's convenience, we provide a short proof of Block and Fang's result here. For that purpose we introduce the following notations.

Let $X_{1}, X_{2}, \ldots, X_{n}$ be random variables and denote by $F\left(t_{1}, t_{2}, \ldots, t_{n}\right)=\mathbf{P}\left(X_{1} \leq\right.$ $\left.t_{1}, X_{2} \leq t_{2}, \ldots, X_{n} \leq t_{n}\right)$ their joint distribution function. More generally, for a subset $I \subseteq\{1,2, \ldots, n\}$ denote

$$
F_{I}\left(\left(t_{i}\right)_{i \in I}\right)=\mathbf{P}\left(X_{i} \leq t_{i}: i \in I\right) .
$$

Define iterated integrals by the recursion

$$
\begin{aligned}
F^{[0,0, \ldots, 0]}\left(x_{1}, x_{2}, \ldots, x_{n}\right) & =F\left(x_{1}, x_{2}, \ldots, x_{n}\right) \\
F^{\left[k_{1}, k_{2}, \ldots, k_{i}+1, \ldots, k_{n}\right]}\left(x_{1}, x_{2}, \ldots, x_{n}\right) & =\int_{-\infty}^{x_{i}} F^{\left[k_{1}, k_{2}, \ldots, k_{i}, \ldots, k_{n}\right]}\left(x_{1}, x_{2}, \ldots, t_{i}, \ldots x_{n}\right) d t_{i}
\end{aligned}
$$

Then one can show by induction that

$$
F^{\left[k_{1}, k_{2}, \ldots, k_{n}\right]}\left(x_{1}, x_{2}, \ldots, x_{n}\right)=\mathbf{E} \frac{\left(x_{1}-X_{1}\right)_{+}^{k_{1}}}{k_{1} !} \frac{\left(x_{2}-X_{2}\right)_{+}^{k_{2}}}{k_{2} !} \cdots \frac{\left(x_{n}-X_{n}\right)_{+}^{k_{n}}}{k_{n} !}
$$

Theorem $5.1([3])$. For any $n \geq 2$ the $n$-th multivariate cumulant is given by

$$
\kappa_{n}\left(X_{1}, X_{2}, \ldots, X_{n}\right)=(-1)^{n} \iint_{-\infty}^{\infty} \ldots \int \sum_{\pi \in \Pi_{n}} F_{\pi}\left(t_{1}, t_{2}, \ldots, t_{n}\right) \mu\left(\pi, \hat{1}_{n}\right) d t_{1} d t_{2} \ldots d t_{n}
$$

where for any partition $\pi \in \Pi_{n}$ we denote

$$
F_{\pi}\left(t_{1}, t_{2}, \ldots, t_{n}\right)=\prod_{B \in \pi} F_{B}\left(t_{i}: i \in B\right)
$$

Proof. Assume first that the random variables $X_{i}$ are bounded from above and choose upper bounds $x_{i}$. Then the subscripts in (5.3) disappear and we have

$$
F^{[1,1, \ldots, 1]}\left(x_{1}, x_{2}, \ldots, x_{n}\right)=\mathbf{E}\left(x_{1}-X_{1}\right)\left(x_{2}-X_{2}\right) \cdots\left(x_{n}-X_{n}\right) ;
$$

because of translation semi-invariance we may use the modified moment-cumulant formula

$$
\begin{aligned}
\kappa_{n}\left(X_{1}, X_{2}, \ldots, X_{n}\right) & =(-1)^{n} \kappa_{n}\left(x_{1}-X_{1}, x_{2}-X_{2}, \ldots, x_{n}-X_{n}\right) \\
& =(-1)^{n} \sum_{\pi \in \Pi_{n}} F_{\pi}^{[1,1, \ldots, 1]}\left(x_{1}, x_{2}, \ldots, x_{n}\right)
\end{aligned}
$$


where $F_{\pi}^{[1,1, \ldots, 1]}\left(x_{1}, x_{2}, \ldots, x_{n}\right)=\prod_{B \in \pi} F_{B}^{[1,1, \ldots, 1]}\left(x_{i}: i \in B\right)$. Writing this out in terms of the recursion (5.2) and noting that the result does not depend on the choice of the bounds $x_{i}$ yields the claimed formula.

Second Proof of Theorem 4.3. If $X_{1}=X_{2}=\cdots=X_{n}$ then the integrand

$$
\sum_{\pi \in \Pi_{n}} F_{\pi}\left(t_{1}, t_{2}, \ldots, t_{n}\right) \mu\left(\pi, \hat{1}_{n}\right)
$$

is symmetric in $t_{1}, t_{2}, \ldots, t_{n}$, we may "shuffle" the integration variables and get

$$
\kappa_{n}\left(X_{1}, X_{2}, \ldots, X_{n}\right)=(-1)^{n} n ! \iint_{-\infty<t_{1}<t_{2}<\cdots<t_{n}<\infty} \ldots \sum_{\pi \in \Pi_{n}} F_{\pi}\left(t_{1}, t_{2}, \ldots, t_{n}\right) \mu\left(\pi, \hat{1}_{n}\right) d t_{1} d t_{2} \ldots d t_{n}
$$

and now observing that the joint distribution function of any subset satisfies

$$
F_{I}\left(t_{i}: i \in I\right)=F\left(\min \left(t_{i}: i \in I\right)\right)
$$

we arrive at the claimed formula.

\section{REFERENCES}

[1] Richard E. Barlow and Frank Proschan, Mathematical theory of reliability, With contributions by Larry C. Hunter. The SIAM Series in Applied Mathematics, John Wiley \& Sons Inc., New York, 1965. MR MR0195566 (33 \#3765)

[2] Bruno Bassan, Michel Denuit, and Marco Scarsini, Variability orders and mean differences, Statist. Probab. Lett. 45 (1999), no. 2, 121-130. MR MR1718455 (2000j:60016)

[3] Henry W. Block and Zhao Ben Fang, A multivariate extension of Hoeffding's lemma, Ann. Probab. 16 (1988), no. 4, 1803-1820. MR MR958217 (90a:62133)

[4] Kuo-Tsai Chen, Integration of paths, geometric invariants and a generalized Baker-Hausdorff formula, Ann. of Math. (2) 65 (1957), 163-178. MR MR0085251 (19,12a)

[5] Wassily Höffding, Masstabinvariante Korrelations-Theorie, Schriften Math. Inst. Univ. Berlin 5 (1940), 181-233.

[6] M. C. Jones, On some expressions for variance, covariance, skewness and L-moments, J. Statist. Plann. Inference 126 (2004), no. 1, 97-106. MR MR2090688 (2005i:62039)

[7] M. C. Jones and N. Balakrishnan, How are moments and moments of spacings related to distribution functions?, J. Statist. Plann. Inference 103 (2002), no. 1-2, 377-390, C. R. Rao 80th birthday felicitation volume, Part I. MR 1897001

[8] E. L. Lehmann, Some concepts of dependence, Ann. Math. Statist. 37 (1966), 1137-1153. MR MR0202228 (34 \#2101)

[9] Rimhak Ree, Lie elements and an algebra associated with shuffles, Ann. of Math. (2) 68 (1958), 210-220. MR MR0100011 (20 \#6447)

[10] G.-C. Rota, Twelve problems in probability no one likes to bring up, Algebraic combinatorics and computer science, Springer Italia, Milan, 2001, pp. 57-93. MR 1854475

Institut für Mathematische Strukturtheorie, TU Graz,, Steyrergasse 30, 8010 Graz, Austria

E-mail address: lehner@finanz.math.tu-graz.ac.at 